\title{
Intramuscular Hematoma Following Radial Extracorporeal Shockwave Therapy for Chronic Neurogenic Heterotopic Ossification: A Case Report
}

\author{
Howard Kim, MD, Ji Hwan Cheon, MD, Dong Youl Lee, MD, Ji Hong Cheon, MD, \\ Youn Kyung Cho, MD, Sung Hoon Lee, MD, Eun Young Kang, MD
}

Department of Rehabilitation Medicine, Kwangju Christian Hospital, Gwangju, Korea

\begin{abstract}
Extracorporeal shockwave therapy (ESWT) has been reported to be a safe and effective method for decreasing pain and relieving range of motion (ROM) limitations caused by neurogenic heterotopic ossification (NHO), though there has been no report that it might cause hematoma if applied to NHO. We hereby report a case of massive hematoma after ESWT, specifically the radial shockwave therapy (RSWT) device at both hips in a 49-yearold female patient with NHO. She had developed NHO after extensive subarachnoid hemorrhage. We had applied RSWT according to the previous report. The pain and the ROM limitations were gradually improved. Six weeks later, she reported pain and ROM limitations on the right hip. From a medial aspect, swelling and bruising of the right thigh could be seen. Magnetic resonance imaging and ultrasonography suggested a large hematoma between right hip adductor muscles. The symptoms disappeared after conservative treatment for one month, and subsequent follow-up imaging studies demonstrated resolution of the hematoma.
\end{abstract}

Keywords High-energy shockwaves, Hematoma, Heterotopic ossification

\section{INTRODUCTION}

Neurogenic heterotopic ossification (NHO) is a potential sequela of neurological disorders that occurs especially after central nervous system (CNS) injury. It is characterized by the formation of new extra-osseous (ectopic) bone in soft tissue surrounding the peripheral joints in patients with neurologic disorders. NHO causes significant morbidity, including pain and loss of motion, especially around joints. In addition, the development of

Received May 9, 2016; Accepted July 5, 2016

Corresponding author: Eun Young Kang

Department of Rehabilitation Medicine, Kwangju Christian Hospital, 37 Yangnim-ro, Nam-gu, Gwangju 61661, Korea. Tel: +82-62-650-5167, Fax: +8262-671-5116, E-mail: eykang74@hanmail.net

ORCID: Howard Kim (http://orcid.org/0000-0002-1123-9485); Ji Hwan Cheon (http://orcid.org/0000-0001-5921-3084); Dong Youl Lee (http://orcid. org/0000-0002-5740-1468); Ji Hong Cheon (http://orcid.org/0000-0003-4366-1610); Youn Kyung Cho (http://orcid.org/0000-0003-4906-6089); Sung Hoon Lee (http://orcid.org/0000-0002-2071-7936); Eun Young Kang (http://orcid.org/0000-0001-6805-5727).

@ This is an open-access article distributed under the terms of the Creative Commons Attribution Non-Commercial License (http://creativecommons.org/ licenses/by-nc/4.0) which permits unrestricted noncommercial use, distribution, and reproduction in any medium, provided the original work is properly cited. Copyright $\odot 2017$ by Korean Academy of Rehabilitation Medicine 
NHO increases the risk of complications caused by immobility, which can significantly impair quality of life. Therefore, it seems desirable to find ways of either preventing or treating this pathological process for improved rehabilitation.

Extracorporeal shockwave therapy (ESWT) is an intense, but short energy wave that travels faster than the speed of sound. There are two principal modalities of shockwave therapy: focused (FSWT) and radial (RSWT). FSWT produces waves faster than the sound velocity, whereas RSWT does not. In contrast to FSWT, RSWT creates a diverging pressure field, which reaches a maximal pressure already at the source. Therefore, it has a more superficial, but broader effect than FSWT. For these reasons, only FSWT was defined as a genuine shockwave treatment, though both FSWT and RSWT have been applied to a growing number of tendon, joint, and muscle conditions with no proven superiority of one modality over the other [1]. In addition, RSWT gradually earns more affordability for calcified lesions [2].

There are several cases reported, where ESWT was effective in relieving pain caused by mature NHO [3-5]. Among these cases, only one of them applied RSWT while the others used FSWT. It was hypothesized that soft tissues around NHO were compressed by calcifying mass and this leads to local ischemic injury. After applying FSWT, it is generally known that soft tissues around NHO has been regenerated from ischemia for several reasons like FSWT stimulating angiogenesis (new blood vessels) and neurogenesis (new nerve cells) [5]. However, there have been no clear explanations about the effects of RSWT on NHO.

The basic mechanism of FSWT originates from extracorporeal shockwave lithotripsy (ESWL), a technology that uses shockwaves to break up kidney stones without surgery. This treatment has been successfully applied for more than 30 years. It was deemed to be a non-invasive and safe treatment, though the occurrence of sub-clinical hematomas of the kidney was estimated to be $15 \%-30 \%$, whereas clinically significant hematoma formation after ESWL was reported in $1 \%$ of the cases in the literature. As generally known, the mechanism of hematoma formation by ESWL is related to the difference in impedance between the renal capsule and parenchyma, and thin arcuate veins in the corticomedullary junctions are vulnerable to shockwaves. Furthermore, while applying FSWT, side effects like petechia, bleeding or hematoma were reported in the magnitude of $4.5 \%$ [6], and even $78 \%$ of small hematomas were manifested following FSWT applied to calcifying lesions [7].

Contrariwise, no significant hematoma cases have been reported with RSWT, but there are accounts of minor side effects like redness of the skin, slight swelling, and discomfort over the treatment area. Moreover, to the best of our knowledge, there are no prior reports of hematoma formation after the use of either FSWT or RSWT as treatments for chronic painful NHO. Here, we report the case of a patient with intramuscular hematoma after RSWT applied to chronic NHO.

\section{CASE REPORT}

A 49-year-old female with no notable medical history was transferred to our hospital for the evaluation of comatose mentation on February 7, 2014. On admission, computed tomography (CT) angiography of the neck and brain were performed subsequently. The examination revealed extensive subarachnoid hemorrhage (SAH) which resulted from a ruptured lobulated aneurysm at tip of the basilar artery. The patient had received aneurysm clipping, and regained consciousness after intensive care for one month. In May 2014, it was noted that she had a developing $\mathrm{NHO}$ around both hips, which resulted in a decreased range of motion, and pain around the hips with a consequent loss of functions. The patient was a severe spastic quadriplegic and needed total assistance for bed-to-wheelchair transfers. The Modified Ashworth Scale of both lower extremities was not assessed due to pain, which made it impossible for her to sit in a wheelchair for a short time. When pain levels were measured on the visual analogue scale (VAS), it was initially 5 and sometimes increased to 7 . The manual muscle testing (Medical Research Council [MRC]) score was $1 / 5$ in all four extremities.

In May 2015, at the time of the intervention, etidronate disodium (Dinol; Chodang Pharmaceuticals, Seoul, Korea) and non-steroidal anti-inflammatory drugs (NSAID) had been administered for 6 months though, neither the size of the NHO had decreased nor had the pain been relieved. Serum alkaline phosphatase (ALP) levels had almost normalized and no further progression of NHO was noted. Therefore, we decided to apply RSWT on chronic 
NHO according to previous reports [4,5]. Etidronate disodium and NSAID had been discontinued one week prior to RSWT. While using MP200 (Storz Medical AG, Tagerwilen, Switzerland) (Fig. 1A), the applicator is slowly moved around a lesser trochanter (Fig. 1B), and transmission gel is applied between the applicator and the skin. Each application of RSWT involved 2,500 shocks delivered at a rate of $12 \mathrm{~Hz}$ with an energy of $3.0 \mathrm{bar}$ ( $1 \mathrm{bar}=0.1$ $\mathrm{MPa}=0.1 \mathrm{~N} / \mathrm{mm}^{2}$ ). All measurements (pain levels, range of motion $[\mathrm{ROM}], \mathrm{ALP})$ were taken immediately after the administration of RSWT and at weekly intervals throughout the 6 -week intervention. Goniometric measurements of both hips' movements were taken with the patient in a supine position, and other physical modalities were not performed, except for pain-free passive ROM exercises.

Following the first RSWT application, the pain was reduced from 5 to 3 on the VAS. Only 4 weeks after the treatment, the pain gradually decreased to the level of 0 and this was maintained until the end of the 6 th session. The ALP levels did not stray from the normal range during the 6-week trial. Comparison of pre- and post-treat- ment X-rays displayed radiographic changes of slightly decreased NHO (Fig. 2A, 2B). The manual muscle testing score revealed no remarkable advancement, however considerable improvement was evidenced for the passive ROM of both hip joints (Table 1).

Only a day after the last successful 6th RSWT, the patient reported pain and passive ROM limitations of the right hip. From a medial aspect, swelling and bruising of the right thigh could be seen (Fig. 3A). The patient had not taken any antiplatelet agents, anticoagulants, or herbal medicine. Her blood pressure was 112/80 mmHg and the other vital signs were inconspicuous. All laboratory values were within normal ranges, including serum creatinine, prothrombin time, and the platelet count.

Magnetic resonance imaging (MRI) and ultrasonography (USG) were taken immediately. There was a large (10 $\mathrm{cm} \times 15 \mathrm{~cm}$ ) heterogeneous high signal intensity on the T1-weighted MRI as well as intermixing of anechoic and hypoechoic portions in an irregular pattern on USG (Fig. 3B, 3C). These findings suggested a large hematoma between the right hip adductor muscles.
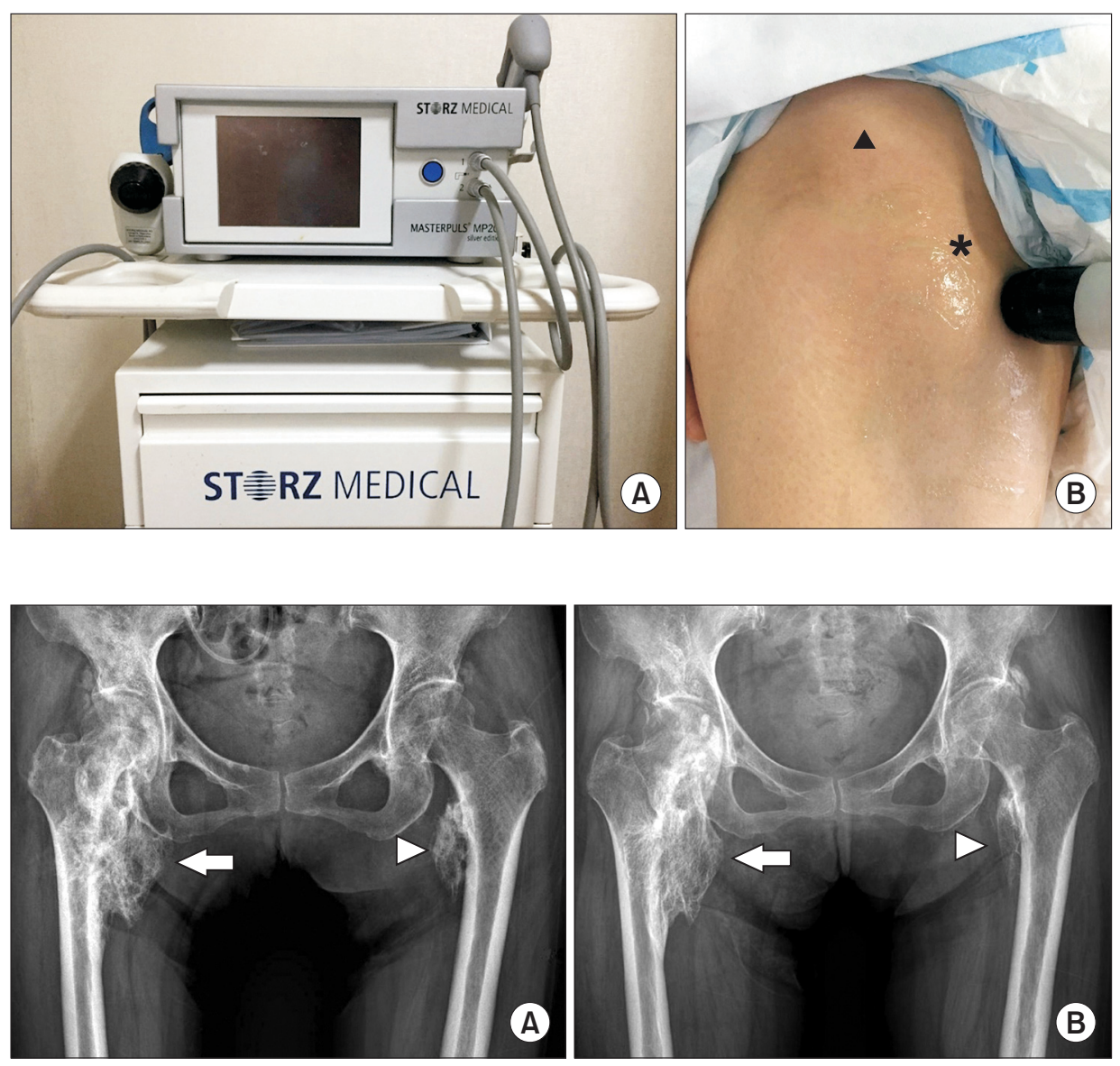

Fig. 1. (A) MP200 (Storz Medical AG, Tagerwilen, Switzerland). (B) Radial shockwave therapy was applied around a lesser trochanter (asterisk) below anterior inferior iliac spine (arrowhead).

Fig. 2. Comparison of X-rays pretreatment (A) and post-treatment (B), which radial shockwave therapy revealed that the size had become slightly smaller in posttreatment on both right (arrow) and left neurogenic heterotopic ossification (arrowhead). 


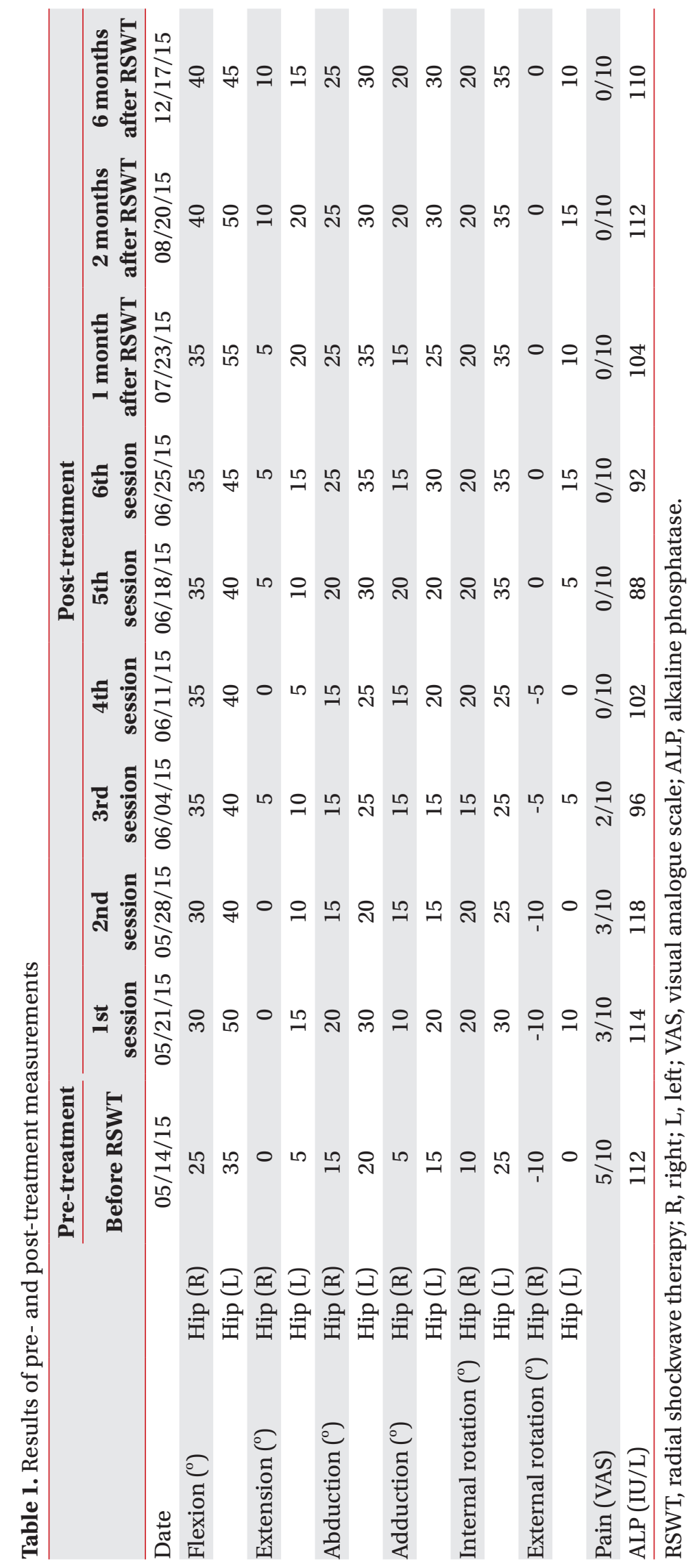




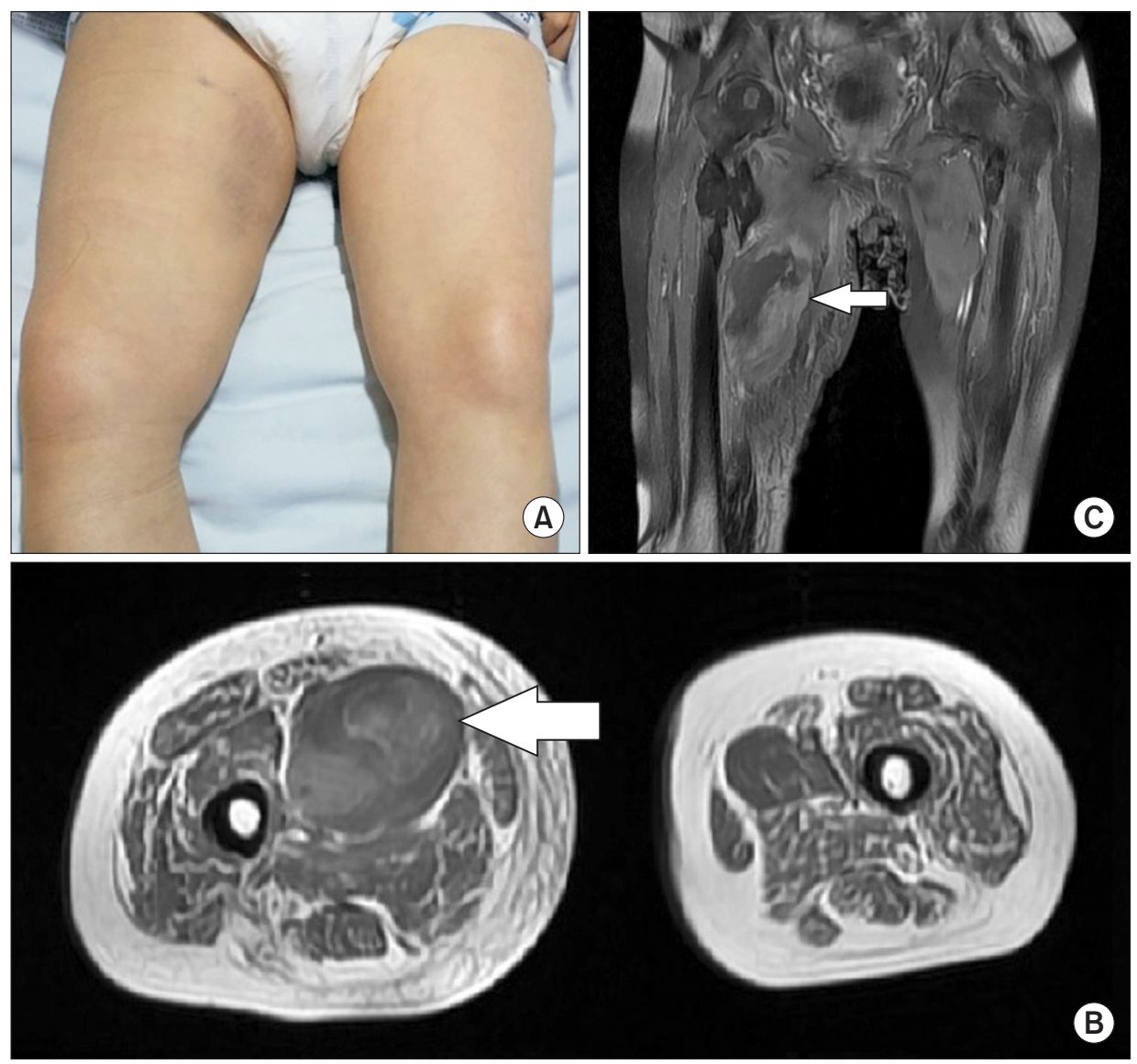

Fig. 3. (A) From a medial aspect of the right thigh, swelling and bruising were seen. Axial (B) and coronal (C) T1-weighted magnetic resonance images show an area of heterogeneous high signal intensity in the right pectineus muscle, consistent with hematoma (arrow).
Fortunately, manual muscle testing of the lower extremities revealed no aggravation and the patient did not experience sensory disturbances. However, the pain had continued several days despite pain relief treatment. After one month of conservative therapy according to the PRICE (protection, rest, ice, compression, and elevation) principle, the bruising, swelling, and pain almost disappeared (Fig. 4A) without any apparent signs of a compartment syndrome. A follow-up MRI and USG also revealed that the hematoma had decreased in size (Fig. 4B, 4C). The level of hip pain maintained 0 until the 18 weeks after RSWT (Table 1). The patient did not articulate any complaints during ROM exercise and training.

\section{DISCUSSION}

Shockwaves may induce the ingrowth of new vessels and tissue proliferation associated with the early release of angiogenesis-related factors including nitric oxide (NO) and the vascular endothelial growth factor. The neovascularization may lead to an improved blood sup- ply and it could play a role in tissue regeneration. The local blood supply increased by shockwaves around NHO may lead to the restoration of oxygen, growth factors, and nutrients, thus causing the hypoxic tissue to return to an aerobic metabolism. Since its introduction in 1980, shockwaves have become the most preferred modality for the majority of patients with renal or proximal urethral calculi. In several studies, shockwaves were also adapted to expand their efficacy to musculoskeletal disorders and NHO. Although generally considered to be safe and effective, a few hematoma cases have been reported with shockwaves use [6,7].

The aforementioned mechanisms and effects of shockwaves were often derived from FSWT. The type of applicator used in this study was RSWT, or more accurately, pressure wave therapy. This method differs from FSWT in its electrohydraulic, electromagnetic, and piezoelectric aspects $[1,8,9]$. After all, the energy wave of FSWT is faster than the speed of sound, or $1,500 \mathrm{~m} / \mathrm{s}$, which is the velocity at which the 'shock' of the shockwaves emanate by breaking the sound barrier. In comparison, RSWT travels 

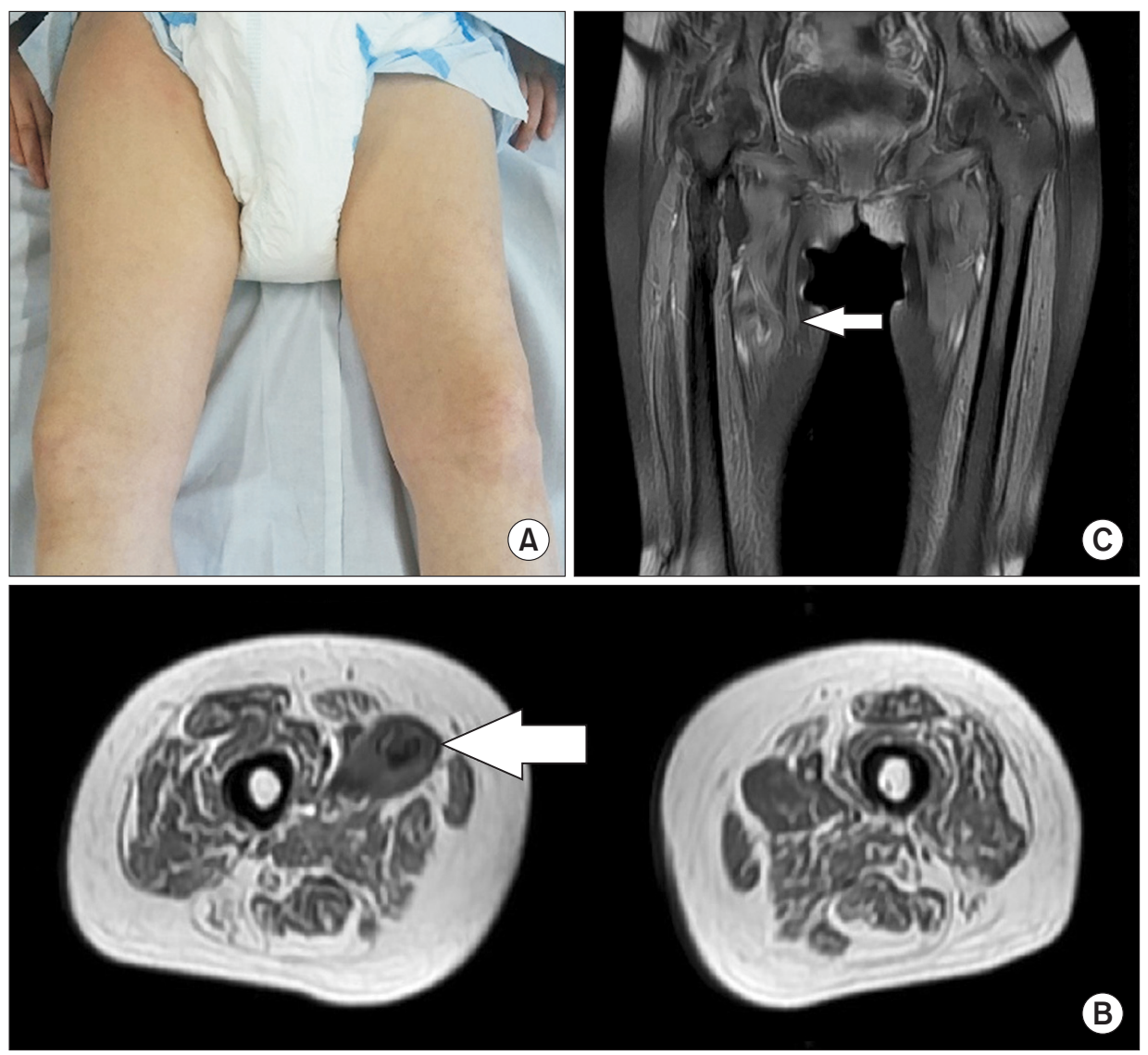

Fig. 4. (A) Swelling and bruising almost disappeared after one month of conservative treatment. Axial (B) and coronal (C) T1-weighted magnetic resonance images show that an area of hematoma had markedly decreased (arrow).

at speeds of approximately $10 \mathrm{~m} / \mathrm{s}$, which is only a small fraction of FSWT. This speed does not break the sound barrier, and hence, no actual shockwaves are produced. Indeed, the very waveform produced by RSWT differs rather noticeably from FSWT. In FSWT, it is very short and highly intense, whereas in RSWT, it is slower, less intense, elongated, and more sinusoidal in appearance.

Intramuscular hematoma can be the consequence of impact against an external blunt or a bone (direct trauma), or of excessive or uncoordinated contraction (indirect trauma). The mechanism of hematoma formation in previous cases caused by true shockwaves (FSWT) has not been elucidated, though it is known to be associated with the piercing effect of a liquid microjet [6-9]. The microjet has an impact velocity of more than $200 \mathrm{~m} / \mathrm{s}$ and occurs as tiny bubbles and the shockwaves interact. Those minute bubbles directly effectuate a loss of vascular tensile strength and they might be a causative factor in hematoma formation. Although it is unclear how tissue damage is initiated, once the hemorrhage occurs, the weight of the blood clot caused by bubbles cuts vessels between the tissues. Thus, the hematoma increases.
Side effects of hematoma formation after shockwaves (ESWL and FSWT) have been explained by the impact of bubbles that are only produced subsequent to the 'shock' breaking the sound barrier. For that reason, RSWT has been generally known as a safe modality towards massive bleeding, because its shockwaves do neither break the sound barrier nor do it generate bubbles to aggravate hemorrhage. Nonetheless, there were also several in vitro cases proving that RSWT also produces bubbles and cavitations $[8,9]$. Although the non-linearity between the cavitations' output and the devices' pulse frequency and/ or energy settings were not evident, the reports clearly demonstrated that RSWT generated cavitation, which exert consequential therapeutic bioeffects and might have caused serious negative effects on the body. Furthermore, a recent study about RSWT applied to the human sole [10] clearly demonstrated that the collision caused between the projectile and the applicator resulted in a stress wave affecting both objects. This wave was transmitted into the soft tissue in the form of compression-rarefaction pressure waves, which could be sufficient to generate tissue cavitations. Moreover, the pressure generated by RSWT 
fell considerably when the waves propagated into the bone in spite of its acoustic impedance being higher than that of the soft tissue. The pressure drop may be explained as follows: the wave reaching the bone splits into transmitted longitudinal shear waves, carrying sufficient energy to break the vascular wall and/or to aggravate bleeding. The results also demonstrated that multiple pulses might have a cumulative effect through a strained energy accumulation in the tissue close to the bone, like sole [10].

Our case is based on the evidence described above that RSWT also brought on compressional and shear stresses as well as cavitations which might cause and/or aggravate hematoma, especially in regions superficial to the bony tissue like the lesser trochanter overlain by large NHO. Moreover, we assume that the patient's positive result to chronic NHO after RSWT is based on the current hypothesis of cavitation-induced microinjury, leading to neovascularization. While supported by experimental evidence, this is largely due to the fact that the pressure field is generated via RSWT in a non-homogeneous medium such as the human body [10].

This research has a number of limitations. We assumed that RSWT directly injured vascular walls or aggravates hematoma, and that the lesions were very close to the major vessels like the femoral artery and vein though, angiographies (arteriography or venography) were not performed. Moreover, to judge the effect of RSWT on NHO, quantifications like three-dimensional CT were also neglected apart from simple X-ray. Also we applied 2,500 shocks of 3.0 bars for six sessions, which is a higher number of treatment sessions compared to previous studies [4], which might have increased the bleeding risks.

Evidently, our case may not directly and conclusively demonstrate the potential damage to NHO by applying RSWT and the effectiveness of RSWT in chronic NHO. However, this study constitutes an alert that RSWT applied to NHO might cause hematoma, and warrants further investigations with larger samples of NHO patients and more types of applicators. Also future research must meticulously scrutinize ESWT's dose-related risks of causing hematoma.

\section{CONFLICT OF INTEREST}

No potential conflict of interest relevant to this article was reported.

\section{REFERENCES}

1. Chang KV, Chen SY, Chen WS, Tu YK, Chien KL. Comparative effectiveness of focused shock wave therapy of different intensity levels and radial shock wave therapy for treating plantar fasciitis: a systematic review and network meta-analysis. Arch Phys Med Rehabil 2012;93:1259-68.

2. Cacchio A, Paoloni M, Barile A, Don R, de Paulis F, Calvisi V, et al. Effectiveness of radial shock-wave therapy for calcific tendinitis of the shoulder: single-blind, randomized clinical study. Phys Ther 2006;86:672-82.

3. Brissot R, Lassalle A, Vincendeau S, Polard JL, Fouche $\mathrm{M}$, Ninubona D, et al. Treatment of heterotopic ossification by extracorporeal shock wave: 26 patients. Ann Readapt Med Phys 2005;48:581-9.

4. Reznik JE, Milanese S, Golledge J, Biros E, Gordon S, Galea MP. Extracorporeal shock wave therapy as a treatment for heterotopic ossification. Phys Ther Rev 2013;18:300-7.

5. Choi YM, Hong SH, Lee CH, Kang JH, Oh JS. Extracorporeal shock wave therapy for painful chronic neurogenic heterotopic ossification after traumatic brain injury: a case report. Ann Rehabil Med 2015;39:31822.

6. Haake M, Boddeker IR, Decker T, Buch M, Vogel M, Labek G, et al. Side-effects of extracorporeal shock wave therapy (ESWT) in the treatment of tennis elbow. Arch Orthop Trauma Surg 2002;122:222-8.

7. Sistermann R, Katthagen BD. Complications, sideeffects and contraindications in the use of medium and high-energy extracorporeal shock waves in orthopedics. Z Orthop Ihre Grenzgeb 1998;136:175-81.

8. Csaszar NB, Angstman NB, Milz S, Sprecher CM, Kobel P, Farhat M, et al. Radial Shock Wave Devices Generate Cavitation. PLoS One 2015;10:e0140541.

9. Kiessling MC, Milz S, Frank HG, Korbel R, Schmitz C. Radial extracorporeal shock wave treatment harms developing chicken embryos. Sci Rep 2015;5:8281.

10. Alkhamaali ZK, Crocombe AD, Solan MC, Cirovic S. Finite element modelling of radial shock wave therapy for chronic plantar fasciitis. Comput Methods Biomech Biomed Engin 2016;19:1069-78. 